PROCEEDINGS OF THE

AMERICAN MATHEMATICAL SOCIETY

Volume 135, Number 10, October 2007, Pages 3301-3308

S 0002-9939(07)08862-4

Article electronically published on June 21, 2007

\title{
POINCARÉ SERIES ON BOUNDED SYMMETRIC DOMAINS
}

\author{
TATYANA FOTH
}

(Communicated by Mei-Chi Shaw)

\begin{abstract}
We show that any holomorphic automorphic form of sufficiently large weight on an irreducible bounded symmetric domain in $\mathbb{C}^{n}, n>1$, is the Poincaré series of a polynomial in $z_{1}, \ldots, z_{n}$ and give an upper bound for the degree of this polynomial. We also give an explicit construction of a basis in the space of holomorphic automorphic forms.
\end{abstract}

\section{INTRODUCTION}

Let $D=G / K$ be an irreducible bounded symmetric domain in $\mathbb{C}^{n}, n>1$; i.e., $G$ is one of the following Lie groups: $S U(p, q), S O^{*}(2 p), S p(p, \mathbb{R}), S O_{o}(p, 2)$, $E_{6}^{(-14)}, E_{7}^{(-25)}[1]$, and $K$ is a maximal compact subgroup of $G$. Denote by 0 the fixed point of $K$ in $D$. Consider a compact smooth quotient $X=\Gamma \backslash D$, where $\Gamma$ is a discrete subgroup of $G$. Denote by $L$ the canonical line bundle on $X$. Let $k$ be a positive integer, and let $\chi$ be a normalized character on $\Gamma$. Denote by $S_{k}(\Gamma, \chi)$ the space of holomorphic $(\Gamma, \chi)$-automorphic forms of weight $k$ on $D$. It is a finite-dimensional complex vector space. If $k$ is sufficiently large (one can take $k \geq(n+2)^{n+7}$, but this is not the best bound), then $L^{\otimes k}$ is very ample, and the Poincaré map is surjective (Theorem $16.5\left[12\right.$ ); i.e., every element of $S_{k}(\Gamma, \chi)$ is the Poincare series of a holomorphic function $f: D \rightarrow \mathbb{C}$. In this paper we show that the function $f$ can always be chosen as a polynomial in $z_{1}, \ldots, z_{n}$ of degree not higher than $N_{0}=\frac{k^{n} n !}{(2 \pi)^{n}} \operatorname{vol}(X)=k^{n} \int_{X} c_{1}(L)^{n}$ or as a linear combination of weighted Bergman kernels at $\operatorname{dim} S_{k}(\Gamma, \chi)$ points in $D$ which project to $\operatorname{dim} S_{k}(\Gamma, \chi)$ distinct points in general position on $X$ under the covering projection $\pi: D \rightarrow X$. The main results of this paper are Theorems 2.1, 2.2, The general approach is as follows. Denote $N=\operatorname{dim} S_{k}(\Gamma, \chi)-1$. Choose points $p_{0}, \ldots, p_{N}$ in $D$ such that $\pi\left(p_{0}\right), \ldots, \pi\left(p_{N}\right)$ are $N+1$ points in general position on $X$. To each of these points, $p_{j}$, associate the automorphic form $\varphi_{k, p_{j}, \chi}$ with the property $\left(f, \varphi_{k, p_{j}, \chi}\right)=f\left(p_{j}\right)$ for any $f \in S_{k}(\Gamma, \chi)$. Here $(.,$.$) denotes the inner product in S_{k}(\Gamma, \chi)$. The $\operatorname{dim} S_{k}(\Gamma, \chi)$ automorphic forms we obtain this way form a basis in $S_{k}(\Gamma, \chi)$.

Now let us change the approach a little. For $l=\left(l_{1}, \ldots, l_{n}\right)$, where $l_{j}, j=$ $1, \ldots, n$, are nonnegative integers, denote by $\varphi_{k, l, 0, \chi} \in S_{k}(\Gamma, \chi)$ the Poincaré series of the monomial $z_{1}^{l_{1}} \cdots z_{n}^{l_{n}}$. We show that if $\left(f, \varphi_{k, l, 0, \chi}\right)=0$ for all $l$ such that $l_{1}+\cdots+l_{n} \leq N_{0}$, then all partial derivatives $\frac{\partial^{l_{1}+\cdots+l_{n} f}}{\partial z_{1}^{l_{1} \cdots \partial z_{n}^{l_{n}}}}(0), l_{1}+\cdots+l_{n} \leq N_{0}$, are

Received by the editors October 6, 2005 and, in revised form, July 25, 2006.

2000 Mathematics Subject Classification. Primary 32N10; Secondary 32N05, 32N15.

The research of this author was supported in part by NSF grant DMS-0204154. 
zero. This implies that the set $\left\{\varphi_{k, l, 0, \chi} \mid l_{1}+\cdots+l_{n} \leq N_{0}\right\}$ spans $S_{k}(\Gamma, \chi)$. This spanning set for $S_{k}(\Gamma, \chi)$ is, in a sense, associated to the point $\pi(0) \in X$.

Similar statements for $n=1, D=S U(1,1) / U(1) \simeq S L(2, \mathbb{R}) / S O(2)$, are well known and are due to Petersson [20, 7]. They are formulated and proved in terms of standard notions of the theory of Riemann surfaces such as holomorphic $k$-differentials and $k$-Weierstrass points. In particular, every automorphic form in $S_{k}(\Gamma, \chi)$ is the Poincaré series of a polynomial in $z$ of degree not higher than $k(2 g-2)$, where $g$ is the genus of the hyperbolic Riemann surface $\Gamma \backslash D$. If $0 \in D$ is not a $k$-Weierstrass point on the uniformized Riemann surface $X$, then every automorphic form of weight $k$ is the Poincare series of a polynomial in $z$ of degree not greater than $k(2 g-2)-g$.

This circle of ideas for $n=1$ is conveyed, in particular, by the following references: [3], 7], 8], 13], 14, [15], [16. For $n \geq 1$ see [24, where the general case of flag domains is studied (bounded symmetric domains are a small part of this class of homogeneous complex manifolds).

\section{AutomorPhic Forms on BOUndED SYMMETRIC DOMAINS}

2.1. Preliminaries. Let $D=G / K$ be a bounded symmetric domain in $\mathbb{C}^{n}, n>1$ : type I: $G=S U(p, q), K=S(U(p) \times U(q))$,

type II: $G=S O^{*}(2 p), K=U(p)$,

type III: $G=S p(p, \mathbb{R}), K=U(p)$,

type IV: $G=S O_{o}(p, 2), K=S O(p) \times S O(2)$,

type V: $G=E_{6}^{(-14)}, K=S O(10) \cdot S O(2)$,

type VI: $G=E_{7}^{(-25)}, K=E_{6} \cdot S O(2)$

(we follow the notation of [1, Table 1]).

Denote by $d \mu_{L}$ the standard Lebesgue measure on $D \subset \mathbb{C}^{n}$. Recall (see e.g. [11, [17) that the Bergman kernel $K(z, w)$ for $D$ is the kernel of the orthogonal projection from $L^{2}\left(D, d \mu_{L}\right)$ to the closed subspace $\left(L^{2} \cap H\right)(D)$ which consists of holomorphic functions, $K(z, w)=\overline{K(w, z)}, K(z, z)>0$ on $D$, for $\gamma \in G$, $K(\gamma z, \gamma w) J(\gamma, z) \bar{J}(\gamma, w)=K(z, w)$, where $J(\gamma, z)$ is the determinant of the Jacobi matrix of $\gamma: D \rightarrow D$ at $z, K(z, w)$ is holomorphic in $z$, antiholomorphic in $w$, and reproducing on holomorphic functions; i.e.,

$$
\int_{D} K(z, w) f(w) d \mu_{L}(w)=f(z)
$$

for any holomorphic function $f \in L^{2}\left(D, d \mu_{L}\right)$.

Denote $\omega=-\frac{i}{2 \pi} \partial \bar{\partial} \ln K(z, z)$; it is a $G$-invariant Kähler form on $D$. Consider the Hermitian metric on $D$ given by $d s^{2}=2 \sum_{j, m} h_{j m} d z_{j} \otimes d \bar{z}_{m}$, where $h_{j m}=\frac{\partial^{2} \ln K(z, z)}{\partial z_{j} \partial \bar{z}_{m}}$. The Bergman metric on $D$ is $\operatorname{Re}\left(d s^{2}\right)=2 \sum_{j, m} h_{j m} d z_{j} d \bar{z}_{m}$, the associated $(1,1)$ form is $-\frac{1}{2} \operatorname{Im}\left(d s^{2}\right)=2 \pi \omega$, and the volume form is $\frac{1}{n !}(2 \pi \omega)^{n}$.

Define $d \mu_{r}=K(z, z)^{r} d \mu_{L}$, where $d \mu_{L}$ is the standard Lebesgue measure on $D \subset$ $\mathbb{C}^{n}$ and $r \leq 0$. Also denote $d \mu(z)=K(z, z) d \mu_{L}(z)$. Consider the weighted Bergman space $\mathcal{H}_{r}^{2}(D)$ which consists of $\mathbb{C}$-valued functions which are holomorphic in $D$ and belong to $L^{2}\left(D, d \mu_{r}\right)$. The reproducing kernel for $\mathcal{H}_{r}^{2}(D)$ is $c(D, r) K(z, w)^{-r+1}$, where $c(D, r)$ is a positive constant given by the following equality 22.

$$
\frac{1}{c(D, r)}=\int_{D}\left(\frac{K(z, w) K(w, z)}{K(z, z) K(w, w)}\right)^{-r+1} d \mu(w) .
$$


2.2. $\Gamma$-automorphic forms. In this subsection we shall recall necessary facts regarding holomorphic automorphic forms with the canonical automorphy factor.

Let $\Gamma$ be a discrete cocompact subgroup of $G$ which acts freely on $D$. Let $k$ be a positive integer. By definition a holomorphic $\Gamma$-automorphic form of weight $k$ on $D$ is a holomorphic function $f: D \rightarrow \mathbb{C}$ such that

$$
f(\gamma z)[J(\gamma, z)]^{k}=f(z)
$$

for any $\gamma \in \Gamma, z \in D$. Denote by $S_{k}(\Gamma)$ the space of holomorphic $\Gamma$-automorphic forms of weight $k$ on $D$. We have $S_{k}(\Gamma) \simeq H^{0}\left(X, L^{\otimes k}\right)$, and $\pi^{*} s=f(z)\left(d z_{1} \wedge\right.$ $\left.\cdots \wedge d z_{n}\right)^{\otimes k}$, where $X=\Gamma \backslash D, L=\Lambda^{n} T^{*^{\prime}} X$ is the canonical line bundle on $X$, $s \in H^{0}\left(X, L^{\otimes k}\right), f \in S_{k}(\Gamma)$, and $\pi: D \rightarrow X$ is the covering map.

We note that $\pi^{*} c_{1}(L)=[\omega]$. For a sufficiently large $k$ there is a well-defined embedding $X \hookrightarrow \mathbb{C P}^{N} \simeq \mathbb{P}\left(H^{0}\left(X, L^{\otimes k}\right)^{*}\right), p \mapsto\left[s_{0}(p), \ldots, s_{N}(p)\right]$, where $N=$ $\operatorname{dim} H^{0}\left(X, L^{\otimes k}\right)-1$ and $s_{0}, \ldots, s_{N}$ is a basis in $H^{0}\left(X, L^{\otimes k}\right)$.

Recall that, by definition, the Poincaré series of a holomorphic function $f \in$ $L^{1}\left(D, d \mu_{1-\frac{k}{2}}\right)$ is $\sum_{\gamma \in \Gamma} f(\gamma z)[J(\gamma, z)]^{k} \in S_{k}(\Gamma)$. For sufficiently large $k$ the series converges absolutely and uniformly on compact sets (4, Theorem 9.1]) and the Poincaré map

$$
\begin{gathered}
\left(L^{1} \cap H\right)\left(D,\left(\Lambda^{n} T^{*^{\prime}} D\right)^{\otimes k}\right) \rightarrow H^{0}\left(X, L^{\otimes k}\right), \\
s \mapsto \sum_{\gamma \in \Gamma} s(\gamma z),
\end{gathered}
$$

is surjective (see [12, Chapter 7] and references therein).

2.3. $(\Gamma, \chi)$-automorphic forms. In this subsection we shall discuss holomorphic automorphic forms with the canonical automorphy factor twisted by the character $\chi^{k}$ (see e.g. [19, section 10], [22, also [6, 1.2.1]).

Let $\chi: \Gamma \rightarrow U(1)$ be a normalized character on $\Gamma$. Denote by $\operatorname{Pic} \Gamma(D)$ the set of isomorphism classes of holomorphic line bundles on $D$ equipped with a holomorphic action of $\Gamma$ which is compatible with the action of $\Gamma$ on $D$ and is linear on the fibers. We note that there is a well-defined map $\operatorname{Pic}(X) \rightarrow \operatorname{Pic} \Gamma(D),[E] \mapsto\left[\pi^{*} E\right]$. Denote by $P i c^{0}(X)$ the subgroup of $\operatorname{Pic}(X)$ formed by holomorphic line bundles with $c_{1}=0$. Denote by $S_{k}(\Gamma, \chi)$ the space of holomorphic functions $f: D \rightarrow \mathbb{C}$ satisfying the automorphy law

$$
f(\gamma z)[J(\gamma, z) \chi(\gamma)]^{k}=f(z)
$$

for any $z \in D, \gamma \in \Gamma$. The space $S_{k}(\Gamma, \chi)$ is isomorphic to $H^{0}\left(X,(L \otimes E)^{\otimes k}\right)$, where $E$ is a holomorphic line bundle on $X$ which is isomorphic to $D \times \chi_{\chi^{-1}} \mathbb{C}=D \times \mathbb{C} / \sim$, $(z, \xi) \sim(\gamma z, \chi(\gamma) \xi)$ for $z \in D, \xi \in \mathbb{C}, \gamma \in \Gamma$.

The line bundle $L \otimes E$ is ample. Therefore by [12, Theorem 16.5] for $k \geq$ $(n+2)^{n+7}$, the bundle $(L \otimes E)^{\otimes k}$ is very ample and the Poincare map

$$
\left(L^{1} \cap H\right)\left(D, \pi^{*}\left((L \otimes E)^{\otimes k}\right) \rightarrow H^{0}\left(X,(L \otimes E)^{\otimes k}\right)\right.
$$

is surjective.

Denote by $\Gamma^{\prime}$ the commutator subgroup of $\Gamma . T=P i c^{0}(X)$ is a complex torus, $\operatorname{dim} T=b_{1}(X)=r k\left(\Gamma / \Gamma^{\prime}\right)$ which is necessarily zero if $G$ is not $S U(n, 1)$ by [18, Theorem 7]. Ball quotients with nonzero first Betti number do exist; see e.g. [10] for explicit examples. [19, Theorem 4] gives the list of bounded symmetric domains 
for which $\operatorname{Pic}(X)$ is isomorphic to the direct product of $\mathbb{Z}$ and a finite abelian group $\operatorname{Char}\left(\Gamma / \Gamma^{\prime}\right)$.

We note that $\pi^{*} c_{1}(L \otimes E)=\pi^{*} c_{1}(L)=[\omega]$. We shall later need an embedding $\iota: X \rightarrow \mathbb{C P}^{N} \simeq \mathbb{P}\left(H^{0}\left(X,(L \otimes E)^{k}\right)^{*}\right), p \mapsto\left[s_{0}(p), \ldots, s_{N}(p)\right]$, where $N=\operatorname{dim} H^{0}\left(X,(L \otimes E)^{\otimes k}\right)-1$ and $s_{0}, \ldots, s_{N}$ is a basis in $H^{0}\left(X,(L \otimes E)^{\otimes k}\right)$.

2.4. Explicit constructions. For a point $p \in D$ define

$$
\Theta_{k, 0, p, \chi}(z)=\sum_{\gamma \in \Gamma}[K(\gamma z, p) J(\gamma, z) \chi(\gamma)]^{k} \in S_{k}(\Gamma, \chi)
$$

and

$$
\Theta_{k, l, 0, \chi}(z)=\sum_{\gamma \in \Gamma} P_{l}(\gamma z)[J(\gamma, z) \chi(\gamma)]^{k} \in S_{k}(\Gamma, \chi),
$$

where $P_{l}(z)=z_{1}^{l_{1}} \cdots z_{n}^{l_{n}}, l_{j}$ are nonnegative integers, and $l=\left(l_{1}, \ldots, l_{n}\right)$. Functions $K(z, p)$ and $P_{l}(z)$ are bounded continuous functions on $D$. Therefore series (2.1), (2.2) converge absolutely, uniformly on compact sets (by [12, Lemma 5.14]; see also [2, 5.2] or [4, Theorem 9.1] in the case $\chi \equiv 1$ ).

Theorem 2.1. Let $p_{0}, \ldots, p_{N}$ be points in $D$ such that $\iota \circ \pi\left(p_{0}\right), \ldots, \iota \circ \pi\left(p_{N}\right)$ are not on the same hyperplane in $\mathbb{C P}^{N}$. Then the Poincaré series $\Theta_{k, 0, p_{j}, \chi}, j=0, \ldots, N$, form a basis in $S_{k}(\Gamma, \chi)$.

Theorem 2.2. The Poincaré series $\Theta_{k, l, 0, \chi}, 0 \leq l_{1}+\cdots+l_{n} \leq N_{0}$, where $N_{0}=$ $\frac{k^{n} n !}{(2 \pi)^{n}} \operatorname{vol}(X)$, span $S_{k}(\Gamma, \chi)$.

Remarks. 1. The statement of Theorem 2.1 does not depend on the choice of the basis $\left\{s_{j}\right\}$ that was made to define the map $\iota$.

2. In Theorem 2.1 we need $N+1$ points $\pi\left(p_{j}\right), j=0, \ldots, N$, in general position on $X$, i.e., $N+1$ points whose images under $\iota$ are not on the same hyperplane in $\mathbb{C P}^{N}$. This is equivalent to requiring that $\left(v_{0}, \ldots, v_{N}\right) \notin \lambda^{-1}(0)$, where $v_{j} \in V=$ $\mathbb{C}^{N+1}$ are such that

$$
\mu\left(v_{j}\right)=\pi\left(p_{j}\right), \mu: \mathbb{C}^{N+1}-\{0\} \rightarrow \mathbb{C P}^{N}
$$

is the natural map, and

$$
\lambda: V \times \cdots \times V \rightarrow \bigwedge^{N+1} V \cong \mathbb{C},\left(u_{0}, \ldots, u_{N}\right) \mapsto u_{0} \wedge \cdots \wedge u_{N} .
$$

3. Suppose $p^{\prime}=\gamma^{\prime} p$, where $\gamma^{\prime} \in \Gamma, p, p^{\prime} \in D$ and $\pi(p)=\pi\left(p^{\prime}\right) \in X$. Then

$$
\Theta_{k, 0, \gamma^{\prime} p, \chi}(z)=\frac{\chi\left(\gamma^{\prime}\right)^{k}}{\bar{J}\left(\gamma^{\prime}, p\right)^{k}} \Theta_{k, 0, p, \chi}(z) .
$$

Therefore the basis in Theorem 2.1 indeed depends not only on the choice of $N+1$ points in general position on $X$ but also on the choice of points representing them on the universal cover. The Poincaré series (2.1) (and corresponding sections of $(L \otimes E)^{\otimes k}$ as well) associated to two different points in $D$ which represent the same point in $X$ differ by a nonzero constant multiplicative factor. It is also clear that in order to get a basis we do not have to choose the points in $D$ in the same fundamental domain.

4. The statement of Theorem 2.2 can be rephrased as follows: any $f \in S_{k}(\Gamma, \chi)$ is equal to the Poincaré series of a polynomial in $z_{1}, \ldots, z_{n}$ of degree not greater than $N_{0}$. This is, of course, the $n>1$ generalization of the Petersson's observation for the 
classical holomorphic automorphic forms. Note: if $n=1$, then $\operatorname{vol}(X)=2 \pi(2 g-2)$, $N_{0}=k(2 g-2)$.

5. Heuristically (2.1) is the $k$-th component of the delta-function at $\pi(p)$ and (2.2) is a linear combination of the $k$-th components of the delta-function at $\pi(0)$ and its derivatives.

6. The number of elements in the finite spanning set we obtain is equal to the number of monomials in $z_{1}, \ldots, z_{n}$ of degree not greater than $N_{0}$. To see how close this is to $\operatorname{dim} H^{0}\left(X,(L \otimes E)^{\otimes k}\right)$ asymptotically as $k \rightarrow \infty$ we note that $\operatorname{dim} H^{0}\left(X,(L \otimes E)^{\otimes k}\right) \sim \operatorname{const}(X) k^{n}$ and the number of monomials in $z_{1}, \ldots, z_{n}$ of degree not higher than $N_{0}$ (which is equal to the number of monomials in $n+1$ variables of degree $N_{0}$ ) is

$$
\frac{\left(n+N_{0}\right) !}{n ! N_{0} !} \sim \operatorname{const}(X) k^{\left(n^{2}\right)}
$$

7. It is well known that for any points $z^{(1)}, \ldots, z^{(m)}$ in $D$ which represent $m$ distinct points on $X$ there is sufficiently large $k$ and there exist Poincaré series $\theta_{j}(z), j=1, \ldots, m$, of weight $k$ such that the $m \times m$ matrix with entries $\theta_{i}\left(z^{(j)}\right)$ has a nonzero determinant. The proof uses asymptotic representations as $k \rightarrow \infty$, with $m, z^{(1)}, \ldots, z^{(m)}$ fixed. It follows that for such $z^{(1)}, \ldots, z^{(m)}$ there exists a Poincaré series of sufficiently large weight with any preassigned values at these points. For this and other statements in this direction, see [21], 2], also [5].

Normalize the Hermitian inner product in $S_{k}(\Gamma, \chi)$ as follows:

$$
(f, g)=c(D, 1-k) \int_{\Gamma \backslash D} f(z) \overline{g(z)} d \mu_{1-k} .
$$

Let $F$ be a Dirichlet fundamental domain for $\Gamma$.

Lemma 2.3. For any $f \in S_{k}(\Gamma, \chi)$,

$$
\left(f, \Theta_{k, 0, p, \chi}\right)=f(p) .
$$

Proof.

$$
\begin{gathered}
\left(f, \Theta_{k, 0, p, \chi}\right)=c(D, 1-k) \int_{F} f(z) \sum_{\gamma \in \Gamma}[K(\gamma p, z) \bar{J}(\gamma, z) \bar{\chi}(\gamma)]^{k} K(z, z)^{-k} d \mu(z) \\
=c(D, 1-k) \sum_{\gamma \in \Gamma} \int_{\gamma F} f(w) \chi(\gamma)^{k}\left(\frac{K(p, w)}{K(w, w)}\right)^{k} \bar{\chi}(\gamma)^{k} d \mu(w) \\
=c(D, 1-k) \int_{D} f(w)\left(\frac{K(p, w)}{K(w, w)}\right)^{k} d \mu(w)=f(p) .
\end{gathered}
$$

Lemma 2.4. If $f \in S_{k}(\Gamma, \chi)$ and $\left(f, \Theta_{k, l, 0, \chi}\right)=0$ for all $l=\left(l_{1}, \ldots, l_{n}\right)$ such that $l_{1}+\cdots+l_{n}<N^{\prime}$, where $N^{\prime}$ is a positive integer, then all partial derivatives

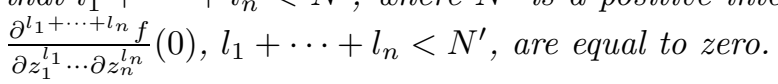


Proof. We have:

$$
\begin{gathered}
\left(f, \Theta_{k, l, 0, \chi}\right)=c(D, 1-k) \int_{F} f(z) \sum_{\gamma \in \Gamma} \overline{P_{l}(\gamma z)}[\bar{J}(\gamma, z) \bar{\chi}(\gamma)]^{k} K(z, z)^{-k} d \mu(z) \\
=c(D, 1-k) \sum_{\gamma \in \Gamma} \int_{\gamma F} f(w) \chi(\gamma)^{k} \overline{P_{l}(w)} \bar{\chi}(\gamma)^{k} K(w, w)^{-k} d \mu(w) \\
=c(D, 1-k) \int_{D} f(w) \overline{P_{l}(w)}(K(w, w))^{-k} d \mu(w) .
\end{gathered}
$$

Now recall that monomials in $z_{1}, \ldots, z_{n}$ form a complete system in $\mathcal{H}_{r}^{2}(D)$ (here we take $r=-k+1$ ), monomials of different degrees are orthogonal to each other, but the inner product of two monomials of the same degree is not necessarily zero [9], 23. Expand $f$ into a series of homogeneous polynomials in $z_{1}, \ldots, z_{n}([23,2.9)$ : $f(z)=\sum_{j=0}^{+\infty} p_{j}(z)$, where $p_{j}(z)$ is of degree $j$. We would like to show that $p_{j}(z)=0$ if $0 \leq j<N^{\prime}$. We obtain:

$$
\left(f, \Theta_{k, l, 0, \chi}\right)=c(D, 1-k) \int_{D} p_{j}(z) \overline{P_{l}(w)}(K(w, w))^{-k} d \mu(w)
$$

where $j=\sum_{m=1}^{n} l_{m}$. By assumption this is zero for all monomials $P_{l}(z)$ of degree $j, 0 \leq j<N^{\prime}$. Therefore $p_{j}=0$ if $0 \leq j<N^{\prime}$.

For domains of type I there is a very explicit relation between the inner product $\left(f, \Theta_{k, l, 0, \chi}\right)$ and the partial derivative $\frac{\partial^{l_{1}+\cdots+l_{n}} f}{\partial z_{1}^{l_{1} \cdots \partial z_{n}^{l_{n}}}}(0)$.

Lemma 2.5. If $D$ is of type $I$, then

$$
\left(f, \Theta_{k, l, 0, \chi}\right)=c(D, 1-k) \frac{1}{\prod_{j=1}^{n} l_{j} !} \frac{\partial^{l_{1}+\cdots+l_{n}} f}{\partial z_{1}^{l_{1}} \cdots \partial z_{n}^{l_{n}}}(0) \int_{D}\left|P_{l}(z)\right|^{2}(K(z, z))^{-k} d \mu(z) .
$$

Proof.

$$
\begin{gathered}
\left(f, \Theta_{k, l, 0, \chi}\right)=c(D, 1-k) \int_{F} f(z) \sum_{\gamma \in \Gamma} \overline{P_{l}(\gamma z)}[\bar{J}(\gamma, z) \bar{\chi}(\gamma)]^{k} K(z, z)^{-k} d \mu(z) \\
=c(D, 1-k) \sum_{\gamma \in \Gamma} \int_{\gamma F} f(w) \chi(\gamma)^{k} \overline{P_{l}(w)} \bar{\chi}(\gamma)^{k} K(w, w)^{-k} d \mu(w) \\
=c(D, 1-k) \int_{D} f(w) \overline{P_{l}(w)}(K(w, w))^{-k} d \mu(w) \\
=c(D, 1-k) \frac{1}{\Pi_{j=1}^{n} l_{j} !} \frac{\partial^{l_{1}+\cdots+l_{n}} f}{\partial z_{1}^{l_{1}} \cdots \partial z_{n}^{l_{n}}}(0) \int_{D}\left|P_{l}(z)\right|^{2}(K(z, z))^{-k} d \mu(z) .
\end{gathered}
$$

The last equality is proved as follows. A domain of type I has symmetries $z_{j} \mapsto$ $e^{i \varphi} z_{j}, \varphi \in \mathbb{R}$. Therefore the equality is true for polynomials in $z_{1}, \ldots, z_{n}$. Then we expand an arbitrary function in $\mathcal{H}_{r}^{2}(D)$ into a series of homogeneous polynomials (with compact convergence) and obtain the desired equality for any function in $\mathcal{H}_{r}^{2}(D)$

Proof of Theorem 2.1. The set $\left\{\Theta_{k, 0, p_{j}, \chi}\right\}$ consists of exactly $N+1=\operatorname{dim} S_{k}(\Gamma, \chi)$ elements. Assume that it is not a basis. Then there is $f \in S_{k}(\Gamma, \chi)$ which is not identically zero and such that $\left(f, \Theta_{k, 0, p_{j}, \chi}\right)=0, j=0, \ldots, N$. Then by Lemma $2.3 f\left(p_{0}\right)=\cdots=f\left(p_{N}\right)=0$. Therefore the corresponding section 
$s \in H^{0}\left(X,(L \otimes E)^{\otimes k}\right)$ vanishes at $\pi\left(p_{j}\right), j=0, \ldots, N$. But $s=\sum_{j=0}^{N} a_{j} s_{j}, a_{j} \in \mathbb{C}$, so the locus in $\iota(X)$ where $s=0$ is the intersection of $\iota(X)$ and a hyperplane in $\mathbb{C P}^{N}$. Since the points $\pi\left(p_{j}\right)$ are, by assumption, not on the same hyperplane, $s$ must be the zero section and hence $f$ is identically zero. This is a contradiction; thus the given set is a basis.

Proof of Theorem 2.2, This will be a proof by contradiction. Assume that the given set does not span $S_{k}(\Gamma, \chi)$. Then there is $f \in S_{k}(\Gamma, \chi)$, not identically zero, such that $\left(f, \Theta_{k, l, 0, \chi}\right)=0$ for all $l=\left(l_{1}, \ldots, l_{n}\right), l_{1}+\cdots+l_{n} \leq N_{0}$. By Lemma 2.4 (with $\left.N^{\prime}=N_{0}+1\right)$ all partial derivatives of $f$ up to the order $N_{0}$ vanish at $z=0$.

Denote by $s$ the section of $(L \otimes E)^{\otimes k}$ that corresponds to $f$. Denote by $V$ the locus in $X$ where $s=0$. We defined $N_{0}=\frac{n ! k^{n}}{(2 \pi)^{n}} \operatorname{vol}(X)$. With our normalization of the volume form (see subsection 2.1) $N_{0}=\int_{X}\left(k c_{1}(L)\right)^{n}$. Note that the value of this integral does not depend on the choice of a 2 -form representing $c_{1}(L)$, and also note that $(L \otimes E)^{\otimes k}=\iota^{*} H$, where $H \rightarrow \mathbb{C P}^{N}$ is the hyperplane bundle. $\iota(V)$ is the intersection of $\iota(X)$ and a hyperplane in $\mathbb{C P}^{N}$. By Poincaré duality $N_{0}=$ $\operatorname{ord}_{V}(s) \int_{V}\left(k c_{1}(L)\right)^{n-1}$, and also we note that $a=\operatorname{ord}_{V}(s)$ and $\int_{V}\left(k c_{1}(L)\right)^{n-1}$ are positive integers. Hence $a \leq N_{0}$, i.e. the order of vanishing of $s$ along $V$ at $\pi(0)$ is not greater than $N_{0}$, and this contradicts the statement that $f$ is not identically zero and all its partial derivatives up to the order $N_{0}$ vanish at $z=0$.

In more detail: the map $\iota \circ \pi$ gives a biholomorphic map from a small neighborhood of 0 in $D$ (say, $U$ ) onto a small neighborhood of $\iota(\pi(0))$ in $\iota(X)$. Thus near 0 $f(z)=(h(z))^{a} g(z)$, where $h, g$ are holomorphic in $U, h(0)=0, g(0) \neq 0$. At least one of the first partial derivatives of $h$ must be nonzero at $z=0$ (because $h$ is the pull-back of a linear function under a biholomorphic map), so $\frac{\partial h}{\partial z_{m}}(0) \neq 0$ for some $m, 1 \leq m \leq n$. Also we observe that all partial derivatives of $h^{a}$ up to the order $a-1$ vanish at $z=0$. Hence there is an $a$-th partial derivative of $f$ which has a nonzero value at $z=0$; indeed,

$$
\frac{\partial^{a} f}{\partial z_{m}^{a}}(0)=a !\left(\frac{\partial h}{\partial z_{m}}(0)\right)^{a} g(0)+0 \neq 0 .
$$

But $a \leq N_{0}$; hence this contradicts Lemma 2.4

Therefore we indeed obtain a spanning set.

\section{ACKNOWLEDGMENTS}

I would like to thank Igor Dolgachev, Alejandro Uribe for discussions, David Borthwick, Ajneet Dhillon, Clifford Earle, Svetlana Katok, János Kollár, Irwin Kra, Peter Sarnak, and Osip Schwarzman - for their comments made by e-mail or in person on different stages of preparation of this paper. I am grateful to the Institut des Hautes Études Scientifiques for hospitality and support. I am thankful to the referee for helpful comments.

\section{REFERENCES}

1. D. Akhiezer, Homogeneous complex manifolds, in Several complex variables IV, Encycl. Math. Sci., Vol. 10, Springer-Verlag, 1990; pp. 195-244. MR1095088 (91i:32001)

2. W. Baily, Introductory lectures on automorphic forms, Iwanami Shoten Publishers and Princeton University Press, 1973. MR0369750 (51:5982)

3. L. Bers, Completeness theorems for Poincaré series in one variable, Proc. Internat. Sympos. Linear Spaces (Jerusalem, 1960), Jerusalem Academic Press, Jerusalem; Pergamon, Oxford, 1961; pp. 88-100. MR0132829(24:A2665) 
4. A. Borel, Introduction to automorphic forms, Proc. Symp. Pure Math. 9 (1966),199-210. MR 0207650 (34:7465)

5. H. Cartan, Fonctions automorphes et séries de Poincaré, J. Analyse Math. 6(1958), 169-175. MR0099450 (20:5889)

6. I. Gelfand, M. Graev, and I. Pyatetskii-Shapiro, Representation theory and automorphic functions, Saunders, 1969. MR0233772 (38:2093)

7. D. Hejhal, Monodromy groups and Poincaré series, Bull. AMS 84(1978), no. 3, 339-376. MR0492237 (58:11383)

8. D. Hejhal, Kernel functions, Poincaré series, and LVA, in In the tradition of Ahlfors and Bers (Stony Brook, NY, 1998), Contemp. Math., 256, AMS, Providence, RI, 2000; pp.173201. MR1759678 (2001e:11042)

9. L. Hua, Harmonic analysis of functions of several complex variables in the classical domains, AMS, 1963. MR0171936 (30:2162)

10. M. Ishida, The irregularities of Hirzebruch's examples of surfaces of general type with $c_{1}^{2}=$ $3 c_{2}$, Math. Ann. 262(1983), no. 3, 407-420. MR692865 (85c:14025)

11. G. Khenkin, The method of integral representations in complex analysis, in Several complex variables I, Encycl. Math. Sci., Vol. 7, Springer-Verlag, 1990; pp. 19-116.

12. J. Kollár, Shafarevich maps and automorphic forms, Princeton University Press, 1995. MR.1341589 (96i:14016)

13. I. Kra, Automorphic forms and Kleinian groups, Math. Lecture Note Series, W.A.Benjamin, Inc., 1972. MR0357775 (50:10242)

14. I. Kra, On the vanishing and spanning sets for Poincaré series for cusp forms, Acta Math. 153(1984), no. 1-2, 47-116. MR744999 (86b:30070)

15. I. Kra, Cusp forms associated to loxodromic elements of Kleinian groups, Duke Math. J. 52(1985), no. 3, 587-625. MR808095 (87i:30084)

16. I. Kra, Bases for cusp forms for quasi-Fuchsian groups, Ann. Acad. Sci. Fenn. Ser. A I Math. 10(1985), 289-298. MR 802490 (86k:30053)

17. S. Krantz, Function theory of several complex variables, AMS, Providence, RI, 2001. MR 1846625 (2002e:32001)

18. Y. Matsushima, On the first Betti number of compact quotient spaces of higher-dimensional symmetric spaces, Ann. of Math. (2) 75(1962), 312-330. MR0158406 (28:1629)

19. Y. Matsushima, On Betti numbers of compact, locally symmetric Riemannian manifolds, Osaka Math. J. 14(1962), 1-20. MR0141138 (25:4549)

20. H. Petersson, Über Weierstrasspunkte und die expliziten Darstellungen der automorphen Formen von reeler Dimension, Math. Z. 52(1949), 32-59. MR0033356 (11:428b)

21. I. Pyatetskii-Shapiro, Automorphic functions and the geometry of classical domains, Gordon and Breach, 1969. MR0252690 (40:5908)

22. A. Selberg, Automorphic functions and integral operators, in Collected papers, vol. I, 464-468.

23. H. Upmeier, Toeplitz operators and index theory in several complex vaiables, Birkhäuser, 1996. MR1384981 (97f:47022)

24. J. Wolf, Completeness of Poincaré series for automorphic cohomology, Ann. of Math. 109(1979), 545-567. MR.534762 (80m:32035)

Department of Mathematics, University of Western Ontario, London, Ontario N6A 5B7, CANADA

E-mail address: tfoth@uwo.ca 